Cahiers « Mondes anciens »

MONDES

ANCIENS

Histoire et anthropologie des mondes anciens

$1 \mid 2010$

Écritures rituelles

\title{
Inscrire un serment en Grèce ancienne : couper et verser
}

Renée Koch-Piettre

\section{(2) OpenEdition}

Journals

Édition électronique

URL : http://journals.openedition.org/mondesanciens/112

DOI : $10.4000 /$ mondesanciens. 112

ISSN : 2107-0199

Éditeur

UMR 8210 Anthropologie et Histoire des Mondes Antiques

Référence électronique

Renée Koch-Piettre, «Inscrire un serment en Grèce ancienne : couper et verser », Cahiers «Mondes anciens » [En ligne], 1 | 2010, mis en ligne le 05 juillet 2013, consulté le 19 avril 2019. URL : http:// journals.openedition.org/mondesanciens/112; DOI : 10.4000/mondesanciens.112

Ce document a été généré automatiquement le 19 avril 2019.

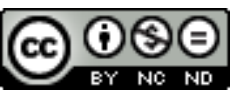

Les Cahiers «Mondes Anciens » sont mis à disposition selon les termes de la licence Creative Commons Attribution - Pas d'Utilisation Commerciale - Pas de Modification 4.0 International. 


\title{
Inscrire un serment en Grèce ancienne : couper et verser
}

\author{
Renée Koch-Piettre
}

\section{Écriture rituelle}

\section{En deçà du pragmatique et du symbolique}

1 L'écriture peut-elle recevoir, dans le rite, une fonction particulière ? Cette fonction nous renseignerait-elle sur une spécificité anthropologique de l'acte d'écrire?

Dans nos élites toujours habitées d'un romantisme qui mariait rite et mythe comme un symbole biface, on demande volontiers aux rites de nous livrer la clef secrète des gestes les plus ordinaires. Comme si le rite recelait le plus archaïque et simultanément le plus profond dans l'homme. Ne pourrait-on se contenter de pointer jusque dans le rite les vertus épistémiques et pragmatiques de l'écriture, pour noter qu'en tout contexte, sacré ou profane, écrire permet de lister, de dénombrer et d'enregistrer (marchandises, offrandes), de marquer et signer (sceau, jeton de sort), de faire mémoire (contrat, pacte, serment), de re-présenter (nomination, inscription, évocation), d'énoncer dans la durée un texte performatif (loi, sentence, invocation), d'agir durablement sur autrui (menace, bénédiction, imprécation) ${ }^{1}$ ? Mais quelque chose résiste : de fait, on n'écrit pas - le geste de l'écriture ne peut exister sans que quelque chose du corps, le plus intime et simultanément le plus nécessaire du corps mû par le désir le plus enfoui et qui échappe au scripteur dans le moment même de son affirmation, ne se projette dans le tracé le plus ténu et le plus prompt à s'effacer. Il doit y avoir dans la nature même de ce geste un impératif étranger à tout à-propos pragmatique.

3 Simultanément, dans sa fonction de représentation, l'écriture permet de faire l'économie de la présence et de l'existence effectives de son référent - tout comme la parole, mais avec une autonomie plus grande parce que rien ne la contraint aux logiques du réel. Indépendante de son référent, elle se suffit à elle-même en ceci que peut-être elle relève 
de cette autre loi que nous venons d'évoquer et qui ressemble à l'Anankè, la " Nécessité ». La parole délirante du fou se heurte au scandale d'autrui et, dans nos sociétés qui ne font aucune place aux réquisitions de cette loi, provoque son internement et le force au mutisme. L'écriture peut au contraire se développer à loisir. La censure même en conserve le produit et l'archive. Ou bien le tag indéchiffrable, aujourd'hui, est promu œuvre d'art (une promotion qui peut-être est la forme suprême d'éviration à défaut d'une autre censure efficace!). Pourtant le tag ne dit souvent rien d'autre que la rage ou l'insolence du scripteur.

4 Nous ne parlerons pas de la fonction symbolique de l'écriture, expression fortement galvaudée. Certes, le symbole aussi a son autonomie, mais on sait également qu'à la différence du signe linguistique il doit être, dans sa forme, motivé ${ }^{3}$. Il est formellement dépendant de ce qu'il symbolise ou des métaphores qui tentent d'approcher un référent même inaccessible. L'écriture, elle, a sa grammaire qui peut inclure l'icône, voire s'y réduire, mais qui peut aussi bien s'en abstraire totalement: voir le cunéiforme ou l'alphabet latin. Elle peut aussi convoquer même à l'aide d'icônes, ad libitum, ce qui n'existe pas ou qui n'existe que par hypothèse (c'est précisément là qu'intervient un usage qu'on peut qualifier de religieux). Par exemple les dieux : on peut en lister à foison, histoire de n'en pas oublier et d'avoir (peut-être) prise, par là même, sur les biens qui d'eux, par hypothèse, dépendent ${ }^{4}$. Ou bien les offrandes aux dieux, vecteurs de la rétribution attendue de leur part: au lieu d'un mouton sacrifié, pourquoi ne pas en inscrire cinq, dix, ou mille? Pourquoi ne pas inscrire un bœuf quand on offre un concombre? Pourquoi, lorsque l'on fait couler, à petits coups, le contenu d'un œuf sur une pierre d'autel, ne pas assigner à chaque fragment de matière oblatoire la valeur d'un poulet entier, un poulet non éclos, un hybride, une chimère, toute une déclinaison de poulets possibles et impossibles inscrits sur un fragment de calebasse en guise d'aidemémoire pour le détail de la dette sacrificielle? Nous ne voulons pas ici parler de l'hyperbole, un phénomène de langue qui n'engage à rien; ni de substitution, d'une offrande du pauvre; mais bien de la remarquable palette d'objets sacrificiels que, dans telle culture d'Afrique subsaharienne, sait offrir l'écriture la plus embryonnaire, et dont elle peut accroitre l'échéancier et stimuler, plutôt que réduire, le paiement ${ }^{5}$. Car si l'enfermement n'arrête pas la main de l'écrivain (ainsi le marquis de Sade), c'est par l'écriture même qu'il tisse une autre prison. Promettre infiniment c'est s'obliger à payer le plus. L'homme se lie en lisant sa dette à mesure qu'il écrit. Le réseau d'internet est à son tour un filet ${ }^{6}$ où se trouve pris et lié tout ce qui de par le monde entier peut être dit et nommé, la nature, l'animal, l'homme et les œuvres de l'homme.

\section{Violence et fluence de l'écriture}

5 Parler d'écriture rituelle, c'est d'autre part mettre en exergue le rôle de l'écriture là même où, dans le domaine religieux, on croit volontiers qu'elle n'a que faire - c'est-à-dire dans les « religions du faire $»^{7}$, au lieu des religions du croire que l'on confond d'ordinaire avec les religions du livre, ces religions chargées d'un enseignement, d'une doctrine, d'une histoire. C'est souligner qu'en matière religieuse le clavier des fonctions pragmatiques de l'écriture en vérité s'enrichit et mérite plus de considération que le rejet trop fréquent de certains gestes scripturaires parmi les manipulations magiques, que l'on tient pour méprisables, si d'aventure on les rencontre au sein des religions du Livre ellesmêmes: tirer au sort des versets sacrés en guise de réponse divinatoire, porter en amulette une formule protectrice en lettres hébraïques, enfouir une tablette de plomb 
chargée d'imprécations, réduire en poudre une page de la Bible pour en fabriquer une médecine, offrir au vent des banderolles chargées de prières...

En-dehors d'une description phénoménologique des occurrences de l'écriture dans le rite, se pose alors la question de la caractérisation formelle du geste d'écrire, de ce faire qu'est l'écrire, parmi la gamme, par ailleurs, des gestes rituels.

7 Si le rite rime avec répétition formelle, alors on peut pointer déjà un premier caractère : l'écriture réduit la multiplicité infinie des formes de la nature, par le moyen du signe, à un nombre limité de traits - et le grec dit stoikheia à la fois les éléments de la nature et les lettres de l'alphabet - dont la répétition codée s'articule au langage pour traduire tout ce qui peut être nommé, imaginé, énoncé. Le nom de rythme y trouve son origine : le ruthmos , mot formé sur la racine de rheô, « couler ", a pu désigner (notamment chez les premiers atomistes grecs) le fluide mouvement qui, d'un petit nombre d'unités graphiques minimales diversement combinées, dessine la forme changeante des lettres 8 .

8 Le geste qui conduit le tracé, nous croyons le connaître d'autre part comme inscription, gravure, incision : le doigt creusant une ligne dans le sable, le stylet griffant la tablette de cire, les pleins appuyés et les déliés de la plume, la pointe de métal gravant l'os, le ciseau entaillant la roche. Il y a là quelque chose qui peut relever de la contrainte et de la violence infligées à la matière, comme est violente la marque imprimée au fer rouge dans la chair humaine ou animale. Une violence que le scripteur s'inflige simultanément à luimême : songeons à l'attitude contrainte du scribe médiéval penché sur son pupitre, aux cahiers d'écoliers encageant entre carrés et lignes droites toutes les velléités d'évasion, aux lettres alignées comme des tableaux de chasse.

Reconnaissons pourtant la fluidité des tracés éphémères sur le sable - imprimés, à l'occasion, par le passage furtif du renard ou de la souris -, ou du geste de la plume courant sur le papier ; la posture nonchalante du scribe persan, le calame parcourant sa page déroulée sur une cuisse levée. Dans l'écriture, le fluent n'est jamais loin de la violence, comme le hasard n'est jamais loin de la contrainte, ni l'arabesque de l'onciale.

10 Nous aimerions relever ici un cas particulier de cette conjonction de la fluence et de la violence de l'écriture dans l'acte rituel. Il s'agit du serment en Grèce ancienne.

\section{Le serment grec}

11 La légende de Faust, relayée par diverses observations ethnographiques, nous a de longue date familiarisés avec l'image du serment écrit avec du sang, ici le propre sang de celui qui s'engage. Nous nous sommes récemment interrogée sur un passage bien connu des

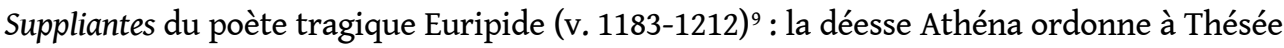
de « recevoir » un serment de non-agression de la part de l'Argien Adraste, d'inscrire ce serment au creux de la cuve d'un trépied de prix où l'on aura fait saigner au cours du rite juratoire trois victimes ovines, et de consacrer ce trépied au foyer d'Apollon, en son sanctuaire delphique. Simultanément, le couteau qui aura servi à faire couler le sang sera enfoui dans la terre près des bûchers funéraires des chefs de la guerre que nous connaissons notamment par les Sept contre Thèbes d'Eschyle. Il fera barrage à toute invasion argienne sur le sol attique, puisqu'il suffira de le déterrer pour jeter l'épouvante parmi l'ennemi.

12 L'écriture paraît ne jouer dans ce traité qu'un rôle d'archivage. Nous suggérons que le sang et le couteau en anticipent le tracé et en construisent la formule opératoire. 


\section{Le couteau, le bâton et le sang} temnein (aoriste tamein) ${ }^{11}$. Un « serment loyal », cela se coupe (horkia pista tamein, « couper les serments loyaux » : dans cette expression horkia désigne à la fois des victimes animales égorgées et le serment ${ }^{12}$ ). Le coutelas (machaira) y joue un rôle esssentiel ${ }^{13}$. Les morceaux coupés qui servent à prêter le serment sont désignés d'un mot formé sur la même racine que temnein: tomia. Les tomia pourront être des pincées de poils tranchés sur la tête de trois agneaux ${ }^{14}$, le sceptre d'Achille ${ }^{15}$, ou des animaux auxquels on tranche la gorge (sphazein) et qu'on dira sphagia: on pourra sphagia temnein, "couper des bêtes en les égorgeant $»^{16}$, mais aussi en tenir dans les mains les morceaux les plus vifs ${ }^{17}$, marcher ou se tenir debout sur la bête découpée ${ }^{18}$, ou passer entre ces morceaux fumants ${ }^{19}$. métaphores du serment. En effet, le serment archaïque était tout entier rite, dont les paroles représentaient l'actualisation nécessaire, en spécifiant le lieu d'application de sa vertu performative. Et le mot «serment » lui-même, horkos, y représentait un objet que l'on pouvait "prendre", "saisir», comme en témoigne encore le verbe proposé par Athéna dans le passage que nous évoquions des Suppliantes d'Euripide: lab' horkon, " prends » ou « reçois un serment ».

Ce sens de "prendre » a également été attribué par certains savants au verbe omnunai ordinairement traduit par « jurer $»^{20}$.

Le chant I de l'Iliade présente un éloquent condensé du serment rituel : Achille jure que le roi Agamemnon se repentira de lui avoir enlevé Briséis, sa " part d'honneur ». Le texte dit exactement : «Je te le déclarerai, et dessus (ou bien : en outre) je saisirai un grand serment (v. 233 : epi megan horkon omoumai)», etc. Ici l'expression « saisir un serment » peut se lire à la lettre, puisque, quelques vers plus loin, les mots "grand serment ", megas horkos s'appliquent au sceptre qu'Achille vient de saisir et qu'il jettera à terre après avoir parlé. De ce serment spécifié comme bâton, il nous est dit qu'il fut coupé (tomên) dans la montagne, que le bronze en ôta tout le feuillage et la possibilité de reverdir pour qu'il devînt aux mains des juges un opérateur du droit maintenu (themistas), et qu'il est d'autre part « traversé de part en part » (peparmenon) de clous d'or. Ce sceptre a donc une histoire, qui commence par le geste de couper; qui se poursuit dans les mains de ceux qui « posent » (tithêmi) les lois; dont les clous sont comme le coin enfoncé de chaque décision prise selon la justice, assortie des malédictions nécessaires contre tout contrevenant; et qui pour finir est, ici, jeté au sol. En heurtant la terre, le bâton va réveiller les instances souterraines garantes de la justice parce qu'elles rétribuent le crime ${ }^{21}$. Mais il est d'abord lui-même une instance active : "le sceptre n'est pas seulement le signe, mais le siège d'une force religieuse ${ }^{22}$; et l'on peut sans conteste identifier cet Horkos au dieu Serment lui-même $\mathrm{e}^{23}$, à condition de bien comprendre que la divinité en question est présente dans l'objet-sceptre comme tel. C'est par le sceptre qu'Achille jure pour introduire son serment, en employant la formule usuelle : nai (« oui ») + accusatif du nom de la divinité prise à témoin ou à partie.

17 Nous admettrons donc, avec Émile Benveniste ${ }^{24}$, que l'expression horkon omnunai, éventuellement condensée en un mot unique, horkômotein, se rapporte d'abord à cette façon de s'emparer, sous une forme concrète, de l'instance elle-même, pour s'y attacher 
et se placer dans son puissant champ de rayonnement et d'action (c'est de la même manière que des suppliants s'attachent à un autel).

Le serment-horkos peut revêtir d'autres formes matérielles: Archiloque (fr. 96 Bergk) s'adresse à qui a "violé ce grand serment (horkon megan), le sel et la table ", autrement dit le lien d'hospitalité, car l'hospitalité engage les hôtes sans qu'il soit besoin qu'ils partagent autre chose que les formes concrètes de cette hospitalité ${ }^{25}$. Aristophane, dans sa comédie Lysistrata, invente une sédition des femmes d'Athènes par la grève du sexe et leur fait chercher le serment qui les liera entre elles; aux questions du vers 183 : «fais voir le serment » (parphaine ton horkon), et du vers 187 : « quel serment jureras-tu ?» (tin' horkon horkôseis ;), la réponse est successivement : « un bouclier renversé », puis « dans le bouclier », puis " un cheval blanc» (v. 185, 188, 191-192). Les conjurées choisissent finalement, en guise de "serment», une grande coupe "renversée $»^{26}$ (v. 195). Leurs diverses réponses pointent les ingrédients matériels de ce que l'on appelle improprement les sacrifices juratoires: un récipient creux pour recevoir le sang, le versement dans ce récipient du sang jaillissant de la gorge incisée, enfin l'animal égorgé, vraisemblablement entendus chacun, par synecdoque, au sens du dispositif entier. On devine simultanément une intention parodique visant la scène du serment dans le bouclier des Sept contre Thèbes 27 .

Dans cette dernière scène, les sept chefs, en prononçant leur serment, touchent de la main le sang (phonou, 45) du taureau égorgé (taurosphagountes, 44) au-dessus d'un bouclier renversé. Dans Lysistrata, les conjurées « sacrifient entièrement » (kathagisô, 238 : en fait, boivent d'un trait) le «sang» (en fait, du vin) d'un pot "égorgé » (mêlosphagousai... stamnion) dans une grande coupe, après avoir prononcé toutes ensemble leur serment, la main sur la coupe. L'Anabase de Xénophon en offre un parallèle : les mercenaires et leur allié perse égorgent (sphaxantes) trois victimes mâles (taureau, verrat, bélier) en faisant couler leur sang dans un bouclier (eis aspida) où ils plongent ensuite (baptontes) leur épée ou leur lance. ${ }^{28} \mathrm{Il}$ s'agit de créer entre les contractants une solidarité puisée dans le sang chaud ou à peine coagulé des victimes, en quoi le parjure menacerait de dissoudre physiquement les contractants.

Or certains emplois d'un composé de temnein, à savoir entemnein, " entailler ", « inciser ", que Chantraine en son dictionnaire étymologique (s.v. $\tau \hat{\varepsilon} \mu v \omega)^{29}$ traduit simultanément par « couper dans, graver, sacrifier, couper des herbes pour un remède », expriment un tracé d'écriture : ainsi, selon les traductions admises, Thémistocle, sur les margelles de fontaines d'eau potable, «fit graver (entamôn)... des inscriptions que les Ioniens purent lire en arrivant le lendemain à l'Artémision ». Hérodote donne le texte, fort long, de ces inscriptions. Mais si l'on pense à une gravure taillée au burin, on peut se demander comment Thémistocle eut le temps de faire exécuter ce travail. Il suffit pourtant de se rappeler les emplois du même verbe en contexte juratoire, pour comprendre qu'il n'y eut pas ici gravure, mais tracé pratiqué à l'aide d'une encre prompte à sécher, par exemple une bouillie, un goudron exprimés d'une vessie, et pourquoi pas le sang d'animaux saignés pour l'occasion. On utilisait ainsi des porcelets afin de purifier un espace rituel (ou politique, l'un n'allant pas sans l'autre) : en pinçant la carotide on pouvait faire couler le sang à mesure sur le pourtour de l'aire. Les bêtes ont donc pu fournir des encriers sur pattes. Par métonymie, dans le sens d'entemnein l'effet (couler) vient ici supplanter la cause (couper), l'épais liquide exprimé l'emporte sur l'incision ${ }^{30}$, et l'on a fort bien pu de cette manière écrire avec du sang. L'emploi d'entemnein en contexte pharmaceutique pourrait s'expliquer de la même façon, comme l'écoulement du suc recherché à partir 
d'une plante incisée. Le Veilleur qui, au prologue de l'Agamemnon d'Eschyle, fredonne pour se tenir éveillé, "fait couler» (entemnôn) un "suc de mélodies, l'antidote du sommeil $»^{31}$.

Il y a donc place pour l'idée de « faire couler » un serment, en l'écrivant avec du sang ${ }^{32}$.

\section{Enclore et répandre}

Cependant l'étymologie la mieux attestée du mot horkos y reconnaît, depuis l'Antiquité, une modulation de la racine de herkos, "enclos », "enceinte ", "barrière ${ }^{33}$, idée qui pouvait se décliner en " prison » (horkanê), « liens » ${ }^{34}$, « scellé » (horkmon), etc. Le serment encercle et lie, il assure le monde entier, par la caution des puissances invoquées ${ }^{35}$.

D'autre part, Jean Bollack ${ }^{36}$ a proposé d'interpréter omnunai non au sens de «saisir », mais au sens d'« invoquer» des témoins. Ainsi les dieux qui jurent par le Styx, éveillant un espace de résonance, invoquent les forces cosmiques dans leur ensemble, telles qu'elles sont enfermées dans les limites du monde, dans cette haute enceinte rocheuse dont le Styx aurait été l'image avant de se transformer en cascade - à moins que son eau ne fût elle-même comprise comme l'élément primordial qui permet de reculer aux limites du monde ${ }^{37}$. Or le Styx est horkos, serment des dieux, en Iliade II, 755 : et ses eaux « terribles » et glacées ${ }^{38}$ dévalent vers les espaces infernaux comme l'image même de la malédiction inséparable d'un serment ${ }^{39}$. Zeus accomplit pour Styx ses promesses (ce dont il s'est fait le fondement et le pilier, hupestê) «de bout en bout, de part en part » (diamperes) ${ }^{40}$, de la même façon que les clous du sceptre d'Achille traversaient de part en part le bâton ${ }^{41}$. Dans l'Hymne homérique à Déméter, c'est «l'eau implacable du Styx » qui est appelée à "savoir ", c'est-à-dire à être le témoin (istô) du serment de Déméter, à lui conférer la validité qui l'enferme en ses propres et infranchissables limites ${ }^{42}$.

Styx évoque ainsi une géographie qui enclôt dans un diamètre horizontal un geste vertical. Achille brandit son sceptre. Des stèles dressées portent inscrite la mémoire des traités. Mais un traité peut se dire aussi spondê, "libation", versement de vin pur ou d'eau qui dessine la verticale inverse, de haut en bas. Quand il s'agit de trancher les querelles entre Immortels qui assurent la stabilité du monde, et de découvrir le menteur qui est à l'origine de la discorde, Zeus envoie chercher, aux limites du monde, dans une aiguière d'or, un peu de l'eau du Styx : celui des dieux qui est fourbe, dès qu'il en fera libation, tombera gisant dans un coma profond et ne réintégrera le banquet des dieux qu'au bout de dix grandes années ${ }^{43}$. Les hauteurs abruptes qu'évoque le Styx sont justement inséparables de cette chute verticale qui se dit (ibid., 786), sur la même racine que la libation, kataleibesthai, "couler vers le bas ", ou bien, sur une racine qu'on a cru pouvoir tirer de la précédente, kateibesthai ${ }^{44}$. Cette verticale, comme celle de la foudre qui s'abat sur la terre (à Olympie, le Zeus Horkios par lequel les athlètes prêtaient serment était représenté menaçant, les deux mains armées de la foudre ${ }^{45}$ ), peut tout aussi bien assurer un centre dans une solide enceinte (tout lieu frappé par la foudre devient inviolable, on le clôt d'une margelle), que dissoudre et brouiller toute limite : rompre un traité, violer un serment, c'est "mélanger en versant », sugchein ${ }^{46}$. Le serment, horkos, et le parjure, epiorkos, sont les deux faces, celle de la promesse et celle de la malédiction qui en (r)éveille l'impact, d'un même geste, d'un rite unique. Un voleur est un habitué du faux serment: horkos. Inversement, Solon a pu employer epiorkos dans le sens positif de serment ${ }^{47}$. 

vertical dans la bouche d'Achille jurant par son sceptre au premier chant de l'Iliade (v. 233). Entre le serment-instance, le serment-clôture, le serment-déversement, il n'y pas contradiction, mais glissement sémiotique, où les signes germent de la dynamique d'un faire ${ }^{48}$. Saisir/invoquer, couper/verser, écrire/enclore/assurer : autant de gestes pour dresser/enfoncer une borne, pour opérer un marquage et y rester attaché, se confondre avec ce mémorial, comme avec la menace même de sa transgression. s'identifier avec la puissance invoquée: l'homme qui s'engage à assassiner Dion de Syracuse, "descend dans le sanctuaire des déesses Thesmophores; après certains sacrifices, il s'enveloppe du manteau pourpre de la déesse et, tenant dans sa main une torche enflammée, il prononce le serment $»^{49}$. Le meurtrier, ici, a revêtu tout l'attirail de la déesse elle-même (Déméter) et se tient comme elle dans son temple.

Une solidarité définitive naît aussi du contact avec le corps palpitant des victimes : le serment de Platées que nous préserve la stèle dite « d'Acharnes » décrit les alliés jurant et prononçant une malédiction « après avoir enseveli (katakalupsantes) les victimes (sphagia) sous leurs boucliers ». Nous comprenons que les soldats ont entassé leurs boucliers audessus des victimes tout juste égorgées, la face concave touchant les chairs sanglantes ${ }^{50}$. Dans cet exemple, les boucliers retournés vers le sol induisent simultanément, pour les contractants, la menace d'une précipitation aux enfers, où descendent les victimes. Ils mettent en place une durée différée, un temps suspendu à la menace de la mort. Le mécanisme de cette solidarité nous est précisé par le texte des serments homériques : au chant III (v. 292-301), Agamemnon égorge trois agneaux, puis :

les couche à terre, palpitants et cherchant un reste de vie (thumou deuomenous) : car le bronze a pris leur force (menos). Alors, puisant le vin au cratère, de leurs coupes ils le déversent d'un coup, en faisant leur prière (...) : «Ô Zeus très glorieux, très grand! et vous tous, dieux immortels! quel que soit celui des deux peuples qui le premier viole ce pacte, tout comme je répands ce vin, que soit répandue à terre (chamadis rheoi) la cervelle de tous les siens (...)

La syntaxe est celle d'une comparaison, mais la performativité du rite et de la malédiction opère une transposition effective, un chassé-croisé, du geste de répandre affectant la matière du rite (le vin, le sang, les entrailles) au malheur appelé sur les parjures (que leur cervelle se répande). Et ce qui rend la malédiction irrévocable, c'est que le vin répandu d'un trait rejoint le sang qui jaillit avec la vie animale qui s'en va, et que le lexique humanise : menos, thumos, sont des instances de la personne humaine, la force et l'ardeur du guerrier, mais ils sont ici, en chassé-croisé, supposés aux agneaux ${ }^{51}$. Le vin, le sang, la cervelle répandus sont déjà ceux des parjures. Ainsi que nous l'avons entrevu plus haut au sujet du sens de "faire couler " reconnu à certains emplois d'un composé de temnein, " couper ", les tomia, matière du sacrifice juratoire qu'Aristophane à l'occasion reconnaît plaisamment dans un pot de vin, doivent être caractérisés par un écoulement: par définition, ils se répandent. Les tomia ne seront donc pas n'importe quels morceaux découpés, les os et les muscles par exemple, mais plutôt les parties molles et fluentes, les viscères, la cervelle ou le sang, voire le vin qui s'y mêle, et qui souligne la liquéfaction recherchée. «Marcher entre les tomia », y compris dans la Septante, c'est passer entre les deux moitiés d'un animal coupé en deux, les entrailles dégoulinantes, ou entre deux bêtes égorgées dont le sang jaillit d'un coup sur les contractants. Une épure de cette performativité en chiasme apparait dans le fameux "Serment des fondateurs" de Cyrène : ce ne sont plus des animaux, mais des figurines de cire - des kolossoi, doubles 
explicites des co-jureurs -, qui fondent au feu au moment même où sont prononcées les imprécations contre les éventuels parjures.

Celui qui ne s'en tient pas à ce serment [et simultanément: qui ne conserve pas la solidité initiale de ces figures déjà en train de fondre], mais qui le transgresse, qu'il fonde et se dissolve, comme fondent ces kolossoi, lui-même et ses descendants et ses biens 52 .

Aux kolossoi figés des morts, doubles amorphes qu'analysait Jean-Pierre Vernant, répondent ces poupées liquéfiées, comme le Serment pétrifié se déverse dans la cataracte du Styx. Près de l'omphalos delphique, le trépied de Thésée garantira une paix solide, mais le tracé du pacte au creux de la cuve suggère la gorge coupée et le déversement du sang qui la fondèrent sur l'effectivité menaçante, différée et déjà là, d'une dissolution ${ }^{53}$. L'incision du burin ou l'encre sèche, faits de l'instrument et de la matière du serment rituel, n'en pérennisent la parenthèse qu'en tant que celle-ci s'est déjà, dès le début refermée.

\section{Conclusion}

De cette brève analyse on retiendra que le rite ne met pas seulement en œuvre une «magie sympathique $»^{54}$, trace ou reste d'un irrationnel culturel pittoresque ou révolu. En vérité toute plume saigne, et le rite juratoire préserve l'évidence opératoire de cette loi, comme un trait fondamental de l'anthrôpos dont quelques-uns de nos exemples ont pu suggérer qu'il s'offre à l'observation bien au-delà des frontières de la Grèce archaïque. L'écriture virtuelle, dans cette perspective, pourrait s'apparenter à la légèreté d'un parjure : on ne s'étonnera pas si son usage coïncide avec une hémorragie sans précédent des forces vives de l'humanité .

\section{BIBLIOGRAPHIE}

Roland BARTHES, Le degré zéro de l'écriture, Paris, Seuil, 1953.

Émile BENVENISTE, « L'expression du serment dans la Grèce ancienne », Revue de l'Histoire des

Religions 135 (1947-1948), p. 92-93.

Émile BENVENISTE, Problèmes de linguistique générale, II, Paris, Gallimard, 1966.

E.J. BICKERMAN, « Couper une alliance », Archives d'histoire du droit oriental 5 (1950-1951), p. 33-156

[repris dans Studies in Jewish and Christian History 1, Leiden 1976, p. 1-32].

J. BOLLACK \& P. JUDET DE LA COMBE, L'Agamemnon d'Eschyle. Le texte et ses interprétations. Agamemnon 1 (“Cahiers de Philologie" 6), Lille 1981.

Catherine BOUANICH, « Des offrandes carnées à l'acte violent. Le rituel de consécration des morceaux choisis à l'époque ptolémaïque comme proposition de corpus », diplôme de l'EPHE, soutenu en novembre 2008 sous la direction de Christiane Zivie-Coche. 
Michel CARTRY, « De la divination au sacrifice : la métaphore de l'attache », dans Michel CARTRY, Jean-Louis DURAND, Renée косн PIETTRE, Architecturer l'invisible. Autels, ligatures, écritures, Turnhout, Brepols, 2009 (BEHE, 138), p. 361-390.

Jean CASABONA, Recherches sur le vocabulaire des sacrifices en grec, des origines à la fin de l'époque classique ("Publications des Annales de la Faculté des Lettres" n.s. 56), Aix-en-Provence 1966.

Pierre CHANTRAINE, Dictionnaire étymologique de la langue grecque, Paris, Klincksieck $1999^{2}$.

M. DILLON, « By Gods, Tongues, and Dogs: The Use of Oaths in Aristophanic Comedy ", Greece \&

Rome, s. 2, 42/2 (1995), p. 135-151.

C.A. FARAONE, « Molten Wax, Spilt Wine and Mutilated Animals : Sympathetic Magic in Near Eastern and Early Greek Oath Ceremonies », The Journal of Hellenic Studies 113, 1993, p. 60-80.

Jean-Jacques GLASSNER, « De l'invention du sacrifice à l'écriture du monde : les repas des dieux en Mésopotamie ", dans Michel CARTRY, Jean-Louis DURAND, Renée KOCH PIETTRE, Architecturer l'invisible. Autels, ligatures, écritures, Turnhout, Brepols, 2009 (BEHE, 138), p. 361-390.

Jack GOODY, La raison graphique. La domestication de la pensée sauvage, trad. fr., Éditions de Minuit, 1979.

Clarisse HERRENSCHMIDT, Les trois écritures. Langue, nombre, code, Paris, Gallimard, 2007.

Renée KOCH PIETTRE, « Un serment gravé dans une cuve », dans Michel CARTRY, Jean-Louis DURAND, Renée KOCH PIETTRE, Architecturer l'invisible. Autels, ligatures, écritures, Turnhout, Brepols, 2009 (BEHE, 137), à paraître.

R. LONIS, « La valeur du serment dans les accords internationaux en Grèce classique », Dialogues d'Histoire Ancienne 6 [1980], p. 267-286.

P. SÁNCHEZ, « Le serment amphictionique [Aeschn. Legat. (2) 115] : un faux du IV siècle ? », Historia 46/2 (1997), p. 158-171.

John SCHEID, Quand faire, c'est croire. Les rites sacrificiels des Romains, Paris, Aubier, 2005.

P. SIEWERT, Der Eid von Plataia, Munich 1972, p. 98-102.

J.-P. VERNANT, Figures, idoles, masques, Paris 1990.

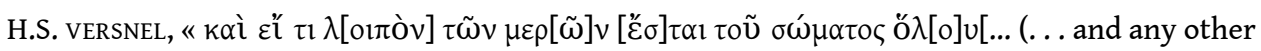
part of the entire body there may be...). An Essay on Anatomical Curses », dans F. GRAF (éd.), Ansichten griechischer Rituale. Geburtstag-Symposium für Walter Burkert, Casteln bei Basel, 15. Bis 18. März 1996, Stuttgart 1998, p. 217-277.

\section{NOTES}

1. Voir Jack GooDy, La raison graphique. La domestication de la pensée sauvage, trad. fr., Éditions de Minuit, 1979.

2. Cette même Loi peut-être de l'«Histoire profonde", où se rencontrent histoire sociale et histoire individuelle, et qui aurait présidé à cette réduction contemporaine de la Littérature au « degré zéro de l'écriture » qu'analysait Roland BARTHEs dans l'ouvrage de ce nom (1953, Paris, Seuil). Mais le souci de Roland Barthes était d'histoire littéraire. Lacan disait, de son côté, que «l'inconscient relève du logique pur, autrement dit du signifiant». Disons donc que cette Loi implacablement « logique » est aussi celle du signifiant, mais on voit aussi que cette logique-là n'a rien de commun avec le logos des philosophes. 
3. À la différence de l'arbitraire du signe dans la théorie de Saussure.

4. On est souvent étonné des énormes quantités d'offrandes et de troupeaux sacrifiés aux dieux de l'Égypte ou de la Mésopotamie anciennes. Ces quantités peuvent parfaitement être imaginaires en tant que nourritures quotidiennes. Ce qui n'en empêche pas l'effectivité occasionnelle ou régulière. Pourquoi dénier, par exemple, à des temples opulents un abattage après tout bien moins massif que dans nos abattoirs industrialisés? Et que dire des abattages annuels dans La Mecque d'aujourd'hui ? Voir Jean-Jacques GLASSNER, « De l'invention du sacrifice à l'écriture du monde: les repas des dieux en Mésopotamie ", dans Michel CARTRY, Jean-Louis DURAND, Renée KOCH PIETTRE, Architecturer l'invisible. Autels, ligatures, écritures, Turnhout, Brepols, 2009 (BEHE, 138) ; pour l'Égypte, signalons un mémoire de Catherine Bouanich pour le diplôme de l'EPHE, soutenu en novembre 2008 sous la direction de Christiane Zivie-Coche, « Des offrandes carnées à l'acte violent. Le rituel de consécration des morceaux choisis à l'époque ptolémaïque comme proposition de corpus ", $1^{\text {re }}$ partie, chap. 1 .

5. Outre le concombre bien connu des Nuer, nous convoquons ici, dans le même volume Architecturer l'invisible, l'article de Michel CARTRY, ethnologue de l'espace Gourmantché : «De la divination au sacrifice: la métaphore de l'attache ». Le lien de foncière équivalence entre la divination, l'écriture et le sacrifice est également développé de façon magistrale par l'ethnologue des Kasena, Danouta LIBERSKI-BAGNOUD, dans un article à paraitre au second volume des Lieux de savoir, publiés par Christian Jacob aux éditions Albin Michel.

6. C'est ce que Clarisse HERRENSCHMIDT nomme «écriture réticulaire » dans son ouvrage récent, Les trois écritures. Langue, nombre, code, Paris, Gallimard, 2007.

7. Voir John SCHEID, Quand faire, c'est croire. Les rites sacrificiels des Romains, Paris, Aubier, 2005.

8. Voir Émile BENVENISTE, Problèmes de linguistique générale, 1, Paris, Gallimard, 1966, p. 327-335.

9. Dans le même volume Architecturer l'invisible, sous le titre « Un serment gravé dans une cuve ». Nous en reprenons ici certaines analyses.

10. Ce trait excède les frontières de la Grèce. Voir E.J. BICKERMAN, « Couper une alliance », Archives d'histoire du droit oriental 5 (1950-1951), p. 33-156 [repris dans Studies in Jewish and Christian History 1, Leiden 1976, p. 1-32].

11. Voir le chapitre sur ce verbe, ses dérivés et ses composés, dans Jean CASABONA, Recherches sur le vocabulaire des sacrifices en grec, des origines à la fin de l'époque classique ("Publications des Annales de la Faculté des Lettres" n.s. 56), Aix-en-Provence 1966.

12. Cf. notamment Iliade III, 252, et toute la scène qui suit.

13. Par ex. Iliade III, 271 ; XIX, 252.

14. Ibid., III, 273. Trois agneaux : 102-104.

15. Voir infra.

16. DÉmosthÈnE, XXIII, 67-68, évoque les tomia des victimes "égorgées» (esphagmenôn). ARISTOPHANE, Lysistrata, 186 et 204, cf. 192 et 196, semble employer tomia et sphagia comme des synonymes.

17. HÉRODOTE, VI, 68.

18. Par exemple DÉMOSTHÈNE, ibid.; PAUSANIAS, III, 20, 9.

19. PlATON, Lois, 753d ; DictYS DE CRÈTE, I, 15 ; II, 49 ; V, 10 (cf. Genèse, 15 ; Jérémie, 34, 18-19).

20. Voir É. BENVENISTE, «L'expression du serment dans la Grèce ancienne », Revue de l'Histoire des Religions 135 (1947-1948), p. 92-93. P. CHANTRAINE le rejoint dans son interprétation (Dictionnaire étymologique de la langue grecque, Paris, Klincksieck $1999^{2}$, s.v.).

21. Voir aussi Iliade VII, 412 : au vers 411, c'est Zeus qui est pris à témoin avec le mot istô, « qu'il voie ", « qu'il sache ", " qu'il soit témoin », et Agamemnon cette fois lève son sceptre vers ce dieu olympien. En XXIII, 581-585, Antiloque est invité à jurer par Poséidon, en touchant ses chevaux, le fouet à la main, parce que l'ardeur du cheval relève de Poséidon. 
22. Voir L.GERNET, Droit et société en Grèce ancienne, Paris 1955, p.17-18 (sur le serment d'Antiloque) ; Id., Anthropologie de la Grèce ancienne, Paris 1995, p. 216, n. 172, sur la conjugaison du sceptre, du geste, de la parole. Notre citation figure p. 205.

23. Cf. HÉSIODE, Théogonie, 231-232: «Serment, le pire des fléaux pour les mortels sur la terre, quand quelqu'un aura, de propos délibéré, commis un parjure » (epiorkon omossêi).

24. É. BENVENISTE, "L'expression du serment », op. cit., p. 92-93. Voir aussi Id., Le vocabulaire des institutions indo-européennes, II, Paris 1969, p. 163-175.

25. Le sel saupoudré crée le lien d'hospitalité, en suscitant l'espace de sa réception (la table) : de la même façon, les projections des grains d'orge sur les participants créent la communauté sacrificielle.

26. Cette coupe présente aux conjurées, comme le bouclier des Sept contre Thèbes, sa face concave remplie de vin, mais si la chose est anormale pour un bouclier, elle ne l'est pas pour une coupe! Les auteurs du dictionnaire LSJ la croient proprement posée à l'envers, mais comment, dans ce cas, contiendrait-elle du vin? L'expression anticipe plutôt le moment où les conjurées boiront d'un trait, la coupe renversée sur leurs lèvres.

27. ESCHYLE, Les Sept contre Thèbes, 43-53. Pour Aristophane, voir M. DILloN, «By Gods, Tongues, and Dogs: The Use of Oaths in Aristophanic Comedy ", Greece \& Rome, s. 2, 42/2 (1995), p. 135-151.

28. Voir aussi le serment des rois atlantes dans le Critias de Platon.

29. Chantraine, $1999^{2}$.

30. HÉRODOTE, VIII, 22-23, voir J. CASABONA, Recherches, op. cit., p. 225. L'historien évoque par le même verbe, au livre $\mathrm{V}, 49$, l'ancêtre de nos cartes de géographie, " une tablette de bronze où étaient gravés (enetetmêto) les contours de toute la terre, toute la mer et tous les fleuves » : selon la technique utilisée, on peut comprendre qu'il s'agit d'une gravure au burin ou - pourquoi pas? - d'un procédé requérant un liquide corrosif, comme pour nos eaux-fortes.

31. ESCHYLE, Agamemnon, 16-17. Traduction de J. BOLLACK, dont nous approuvons le commentaire à ce passage (J. BOLLACK \& P. JUDET DE LA COMBE, L'Agamemnon d'Eschyle. Le texte et ses interprétations. Agamemnon 1 ("Cahiers de Philologie" 6), Lille 1981, p. 22-25).

32. Il n'y aurait donc pas simplement une relation de contiguïté, par magie sympathique (voir làdessus les suggestions de C.A. FARAONE, « Molten Wax », op. cit., p. 60-62; p. 67 pour l'évocation du serment des Suppliantes), mais une homologie des gestes, entre écrire et faire couler le sang.

33. Chez eustathe et dans l'Etymologicum Magnum, cf. R. HIERSCHE, « Note additionnelle », op. cit., p. 39.

34. Le lexicographe HÉsychius donne au pluriel horkoi le sens de « liens, scellés », ou « liens d'un scellé ».

35. Bien entendu, dans les faits, on se parjurait assez vite, et la mémoire ne résistait guère à l'intérêt. Ainsi la garantie du serment

serment

d'alliance a surtout dépendu du rapport des forces (R. LONIS, « La valeur du serment dans les accords internationaux en Grèce classique », Dialogues d'Histoire Ancienne 6 [1980],

p. 267-286).

36. J. BOLLACK, « Styx et serments », Revue des Études Grecques 71 (1958), p. 1-35.

37. Sur Styx : HÉSIODE, Théogonie, 383-403; 775-806. Cf. PAUSANIAS, VIII, 17, 1-6.

38. HÉSIODE, Théogonie, 786.

39. Dans Iliade XV, 38.

40. Ibid., 402sq.

41. Le temps imparti à la Haine dans le système d'Empédocle « s'élance », à la façon d'un attelage dans une carrière, dans les limites que lui assigne " un large serment " (fr. 30, 2-3 dans l'édition de Diels-Kranz) : les liens du serment peuvent être larges jusqu'à enserrer le monde entier, ainsi 
que le rappelle cet autre fragment d'EMPÉDocLE : « Il est une sentence de Nécessité, un décret des dieux, antique, éternel, scellé de larges serments» (fr. 115, 1-2 D.-K.). Ces deux fragments sont cités par J. BOLLACK, «Styx », op. cit., en ouverture à son article.

42. Cf. Odyssée V, 184sq. ; XV, 36sq. ; Hymne homérique à Apollon, 84sq.

43. HÉSIODE, Théogonie, 780-806.

44. Odyssée V, 185 ; Iliade XV, 37.

45. PAUSANIAS, $\mathrm{V}, 24,9$.

46. Par exemple THUCYDIDE, $V, 39,3$, ou les derniers mots du fameux serment d'Hippocrate, évoquant la violation du serment.

47. Odyssée XIX, 396 ; SOLON dans un fragment cité par LYSIAS, 10, 17.

48. "Faire c'est croire » : voir l'exemple romain dans J. SCHEID, Quand faire, c'est croire. Les rites sacrificiels des Romains, Paris 2005.

49. PlutARQue, Dion, 56, 3.

50. Face concave : voir l'analyse de C.A. FARAone, «Molten Wax», op. cit., p. 68 et n. 35. Pour l'historicité de ce témoignage épigraphique, voir aussi la discussion dans P. SIEWERT, Der Eid von Plataia, Munich 1972, p. 98-102, et P. SÁNCHEZ, «Le serment amphictionique [Aeschn. Legat. (2) 115] : un faux du IV ${ }^{\mathrm{e}}$ siècle ?», Historia 46/2 (1997), p. 158-171.

51. Le parallélisme lexical est plus flagrant encore et plus systématique dans un témoignage des paroemiographi graeci cité par C.A. FARAONE, « Molten Wax », op. cit., p. 73.

52. SEG IX, 4. Voir ci-dessus, n. 54. La question des kolossoi a été magistralement traitée par JeanPierre Vernant (textes repris dans J.-P. VERNANT, Figures, idoles, masques, Paris 1990, chap. I à III).

53. L'effectuation rituelle, si sanglante soit-elle, défie une explication par la simple pulsion

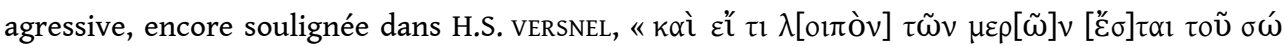
$\mu \alpha \tau \circ \zeta$ ǒ $\lambda[0] \cup[. .$. (...and any other part of the entire body there may be...). An Essay on Anatomical Curses », dans F. GRAF (éd.), op. cit., p. 217-277.

54. Voir le titre de l'article de Faraone, 1993.

\section{RÉSUMÉS}

L'article s'interroge sur une spécificité anthropologique du geste d'écrire que peut révéler le rite du serment dans la Grèce archaïque et classique. Il est introduit par des considérations générales visant à distinguer le geste d'écrire d'une activité simplement langagière et symbolique et à en mettre en valeur deux caractères concrets : selon les techniques employées, l'écriture d'une part appuie, imprime, incise, d'autre part court, déliée et fluide. On montre ensuite que les deux types de gestes peuvent être présents dans des mots de la racine *tem-/*tom- $/{ }^{*}$ tm-, " couper ", qui s'appliquent au rituel juratoire. On « coupe » la gorge des victimes, mais l'important est aussi de faire couler leur sang. Et, avec des coulures, il est possible d'écrire. Entre pacte et dissolution, cette double opération met en place l'efficace de l'engagement du jureur et de la malédiction sur le parjure.

By focusing on the topic of ritual oath in Archaic and Classical Greece, the paper deals with a specific feature of writing as an anthropological gesture. A general introduction aims at the distinction between the ritual gesture of writing and other linguistic and symbolical religious activities; it points out two concrete features of writing: depending on techniques, on the one 
hand hard sealing, engraving or cutting in, on the other hand fluidity. The paper goes on demonstrating that both kinds of gestures appear in the meaning of some Greek words built on the linguistic root *tem- $/{ }^{*}$ tom- $/ *^{*}$ tm- (« to cut »), which applies to ritual oath. People « cut » the sacrificial victim's throat, intending to make blood flowing. Now it is possible to write with drops. From treaty making to pact dissolving, both cutting and dripping set up the efficient commitment of those who swear, and curse on those who break their oath.

INDEX

Mots-clés : écriture, geste, Grèce ancienne, Homère, imprécation, parjure, sang, serment Keywords : Ancient Greece, blood, gesture, Homer, oath, perjury, writing

\section{AUTEUR}

RENÉE KOCH-PIETTRE

EPHE, ANHIMA - UMR 8210 